\title{
Evaluation of the BioFire FilmArray meningitis/encephalitis panel for the detection of bacteria and yeast in Chinese children
}

\author{
Bailu Du ${ }^{1 \#}$, Chunzhen Hua ${ }^{2 \#}$, Yijun $\mathrm{Xia}^{3}$, Jin $\mathrm{Li}^{1}$, Yongping $\mathrm{Xie}^{2}$, Yue Tao ${ }^{4}$, Qing $\mathrm{Cao}^{1}, \mathrm{Xi} \mathrm{Mo}^{4}$ \\ ${ }^{1}$ Department of Infectious Diseases, Shanghai Children's Medical Center, Shanghai Jiao Tong University School of Medicine, Shanghai 200127, \\ China; ${ }^{2}$ Department of Infectious Diseases, The Children's Hospital of Zhejiang University School of Medicine, Hangzhou 310006, China; \\ ${ }^{3}$ Department of Medical Affairs, bioMérieux (Shanghai) Company Limited, Shanghai 201315, China; ${ }^{4}$ The Laboratory of Pediatric Infectious \\ Diseases, Pediatric Translational Medicine Institute, Shanghai Children's Medical Center, Shanghai Jiao Tong University School of Medicine, \\ Shanghai 200127, China \\ Contributions: (I) Conception and design: Y Tao, Q Cao, X Mo; (II) Administrative support: Q Cao, X Mo; (III) Provision of study materials or \\ patients: C Hua, Q Cao; (IV) Collection and assembly of data: B Du, J Li, Y Xie; (V) Data analysis and interpretation: B Du, C Hua, Y Xia; (VI) \\ Manuscript writing: All authors; (VII) Final approval of manuscript: All authors. \\ "These authors contributed equally to this work. \\ Correspondence to: Dr. Xi Mo; Dr. Qing Cao; Dr. Yue Tao. 1678 Dongfang Road, Shanghai Children's Medical Center, Shanghai 200127, China. \\ Email: xi.mo@shsmu.edu.cn; caoqing@scmc.com.cn; taoyue@scmc.com.cn.
}

\begin{abstract}
Background: Meningitis and encephalitis are life-threatening syndromes with high morbidity and mortality in children. Due to limitations of traditional laboratory approaches in etiological diagnosis, the rate of misdiagnoses is unacceptably high.

Methods: We retrospectively compared the potential clinical impact of the FilmArray meningitis/ encephalitis (ME) panel vs. conventional cerebrospinal fluid (CSF) culture in children with central nervous system (CNS) infections. Sixty-eight pediatric patients $(<18$ years of age) with an initial diagnosis of meningitis or encephalitis were enrolled at 2 children's hospital from January to October 2017.

Results: Fifteen specimens were found to be positive after CSF culture, with a positive rate of 22.1\% (15/68). For the FilmArray ME panel, 26 bacteria and fungi from 25 samples were detected, and the positive rate was $36.8 \%$ (25/68). The FilmArray ME panel identified 14 pathogens in previously pathogen-negative patients.

Conclusions: This study demonstrated the capability of the FilmArray ME panel in the diagnosis of bacterial and fungal meningitis and therefore its potential use in facilitating enhanced patient care.
\end{abstract}

Keywords: Meningitis; encephalitis; rapid diagnosis; molecular diagnostic tests; FilmArray ME panel

Submitted Apr 19, 2019. Accepted for publication Aug 20, 2019.

doi: 10.21037/atm.2019.08.103

View this article at: http://dx.doi.org/10.21037/atm.2019.08.103

\section{Introduction}

Meningitis and encephalitis are life-threatening syndromes in children that can be caused by bacteria, yeasts or viruses. The morbidity and mortality of these infections can be high, particularly with bacterial-fungal meningitis. In China, the incidence of acute bacterial meningitis ranges from 6.95 to 22.3 cases/10,000 children $<5$ years of age $(1,2)$. It has been reported that Neisseria meningitidis ( $N$. meningitidis), Haemophilus influenzae (H. influenzae) type $b$ and Streptococcus pneumoniae (S. pneumoniae) are among the most prevalent pathogens in children $(3,4)$. Prompt diagnosis and appropriate antibiotic utilization are necessary to minimize adverse outcomes.

Despite being time-consuming and having low sensitivity (particularly in patients pretreated with antibiotics), routine culture remains the gold standard for the diagnosis of bacterial and fungal meningitis (5). However, the limitations of traditional laboratory approaches lead to unnecessarily prolonged empirical antibiotic treatment and an increase in the number of hospital admissions as well as the duration of 
hospital stays. In addition, the lack of routine diagnostic tests for viral causes of meningitis in China also complicates the problem (6). Rapid, sensitive and comprehensive tests, such as molecular diagnostic tests, may be helpful to overcome the limitations of conventional laboratory-based diagnosis (7).

The FilmArray meningitis/encephalitis (ME) panel (BioFire Diagnostics, Utah, USA, owned by bioMérieux) uses multiplex PCR to detect 14 common pathogens, namely, Escherichia coli K1 (E. coli K1), H. influenzae, Listeria monocytogenes (L. monocytogenes), N. meningitidis, Streptococcus agalactiae (S. agalactiae), S. pneumoniae, cytomegalovirus (CMV), enterovirus (EV), herpes simplex virus 1 and 2 (HSV-1, HSV-2), buman herpesvirus 6 (HHV-6), buman parechovirus (HPeV), varicella zoster virus (VZV), and Cryptococcus neoformans/ Cryptococcus gattii (Cr. neoformans/C. gattii). The entire process is fully automated and takes only approximately one hour to obtain the diagnostic results. The FilmArray ME panel has been increasingly used in many western countries in recent years $(8,9)$. However, its clinical significance in diagnosing ME etiology in the Chinese population is sparse since it is rarely utilized in China. Therefore, in this study, we retrospectively compared the potential clinical impact of the FilmArray ME panel vs. conventional cerebrospinal fluid (CSF) culture in children with suspected or confirmed central nervous system (CNS) infections.

\section{Methods}

\section{Clinical specimens}

This research was a retrospective study. The study was conducted at 2 children's hospitals, namely, Shanghai Children's Medical Center and Zhejiang Children's Hospital, from January 2017 to October 2017. The study was approved by the Institutional Review Board and the Ethics Committee of Shanghai Children's Medical Center (SCMCIRB-K2017059). Written informed consent was obtained from the parents of the participants when lumbar puncture (LP) was carried out.

Pediatric patients ( $<18$ years old) with an initial diagnosis of meningitis or encephalitis were enrolled. Meningitis or encephalitis was defined according to the World Health Organization (WHO) workbook recommendations based on laboratory findings, symptoms, or signs. In addition, all patients were subjected to the following: (I) complete medical history and (II) full clinical examination. Patients were excluded from the study if they met the following criteria: (I) cases complicated with congenital diseases or chronic medical conditions and (II) cases in which other CNS disorders could not be excluded.

For FilmArray ME panel testing, specimens meeting the following inclusion criteria were selected: CSF specimens were collected by LP with adequate volume $(>1 \mathrm{~mL})$; specimens were stored at $-80^{\circ} \mathrm{C}$ for later testing. Duplicate specimens from the same subject were excluded.

\section{FilmArray ME panel testing}

The FilmArray ME panel testing procedure was performed at Shanghai Children's Medical Center according to the manufacturer's instructions. The operation was performed by independent researchers who were blinded to the diagnosis. The test consisted of automated nucleic acid extraction, reverse transcription and nucleic acid amplification. Comprehensive results were available within approximately 1 hour.

\section{Bacterial and fungal conventional testing}

Conventional bacterial and fungal testing programs, including CSF culture, blood culture, Gram strain, ink stain, physiology and biochemistry of CSF, latex agglutination test and serum virology were performed on every subject enrolled. Testing was performed at either hospital using the laboratories' standard procedures.

\section{PCR and sequencing to detect CSF bacterial and fungal infection}

Nucleic acid was extracted from each specimen using a QIAamp DNA minikit (Qiagen, Hilden, Germany). PCR was performed using universal primers for the bacterial 16S rDNA gene and fungal $26 \mathrm{~S}$ rDNA gene. The sequence of the primers used for PCR amplification is provided in Table S1. Pathogens were identified by analyzing DNA sequences using the BLAST tool of NCBI. The results of the PCR analysis were reported as part of the data used for the discrepancy investigation.

\section{Discrepancy analysis}

Samples with discrepant results between the CSF culture and the FilmArray ME panel were reanalyzed using a targeted PCR assay. A FilmArray ME panel result was considered true positive (TP) or true negative (TN) only when it agreed with the results from CSF culture or the 


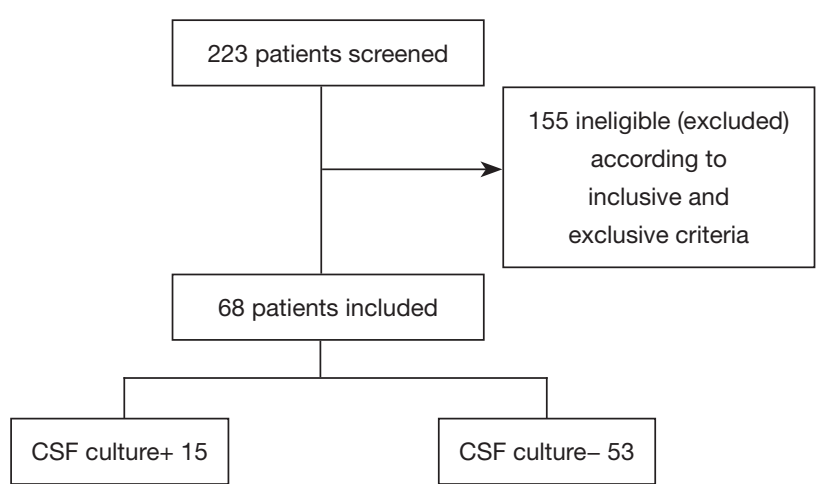

Figure 1 The enrollment process of patients with meningitis or encephalitis.

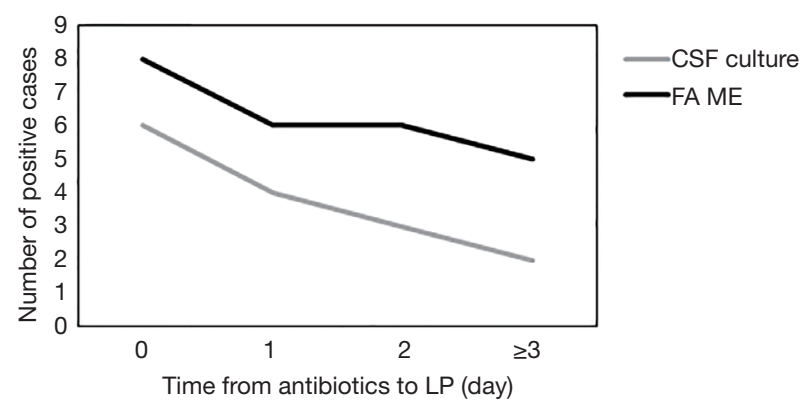

Figure 2 The correlation between the number of days that each patient was on antibiotics before lumbar puncture and the CSF culture and FA ME panel results. CSF, cerebrospinal fluid; FA ME, FilmArray meningitis/encephalitis.

comparator methods. Otherwise, the results were judged as false positive $(\mathrm{FP})$ or false negative $(\mathrm{FN})$.

\section{Data analysis}

Statistical analysis was performed using SPSS ver. 22. Demographic data are presented as descriptive statistics. The agreement between assays was measured using the kappa statistic. The overall percentage of agreement (OPA) was calculated as previously described (10). Briefly, OPA was calculated as $[(\mathrm{TP}+\mathrm{TN}) /(\mathrm{TP}+\mathrm{TN}+\mathrm{FP}+\mathrm{FN})] \times 100 \%$. The sensitivity and specificity were compared for all tests.

\section{Results}

\section{Patient population and clinical feature}

A total of 223 patients were screened, for whom $68 \mathrm{CSF}$
Table 1 General characteristics of the patients

\begin{tabular}{lc}
\hline Characteristic & Cases (\%) \\
\hline Total & $68(100.0)$ \\
Age & $21(30.9)$ \\
$<1$ month & $14(20.6)$ \\
$1-11$ months & $23(33.8)$ \\
$2-6$ years & $10(14.7)$ \\
$7-18$ years & \\
Gender & $46(67.6)$ \\
Male & $22(32.4)$ \\
Female & \\
Antibiotic use before LP & $50(73.5)$ \\
Yes & $18(26.5)$ \\
No & $16(23.5)$ \\
Clinical diagnosis & \\
Bacterial meningitis & $35(51.5)$ \\
Viral encephalitis & $16(23.5)$ \\
Cryptococcal meningoencephalitis & $1(1.5)$ \\
Others & 16 \\
\hline
\end{tabular}

LP, lumbar puncture.

samples met the inclusion criteria between January and October 2017 (Figure 1). The average age of the patients was 2.76 years (range, 3 days to 12 years), and the male/female ratio was 2.09 (46:22). There were 21 (30.9\%) newborns (ages: 1-28 days), 14 (20.6\%) infants (ages: 2-12 months), $23(33.8 \%)$ preschoolers (ages: $2-6$ years) and 10 (14.7\%) school-aged children (ages: $7-18$ years). A total of 50 patients $(73.5 \%)$ in the study had received antibiotic treatment (ceftazidime, meropenem, penicillin, etc.) before LP. The correlation between the number of days that each patient was on antibiotics before LP and the CSF culture and FA ME panel results is illustrated in Figure 2. Thirty-five patients $(51.5 \%)$ were diagnosed with bacterial meningitis, 16 patients $(23.5 \%)$ with viral encephalitis and 1 patient $(1.5 \%)$ with cryptococcal meningoencephalitis. Their general characteristics are presented in Table 1.

\section{Pathogens detected in pediatric patients}

Fifteen specimens were found to be positive after CSF 
Table 2 Distribution of bacteria and yeast identified by the FilmArray ME (FA ME) panel and CSF culture

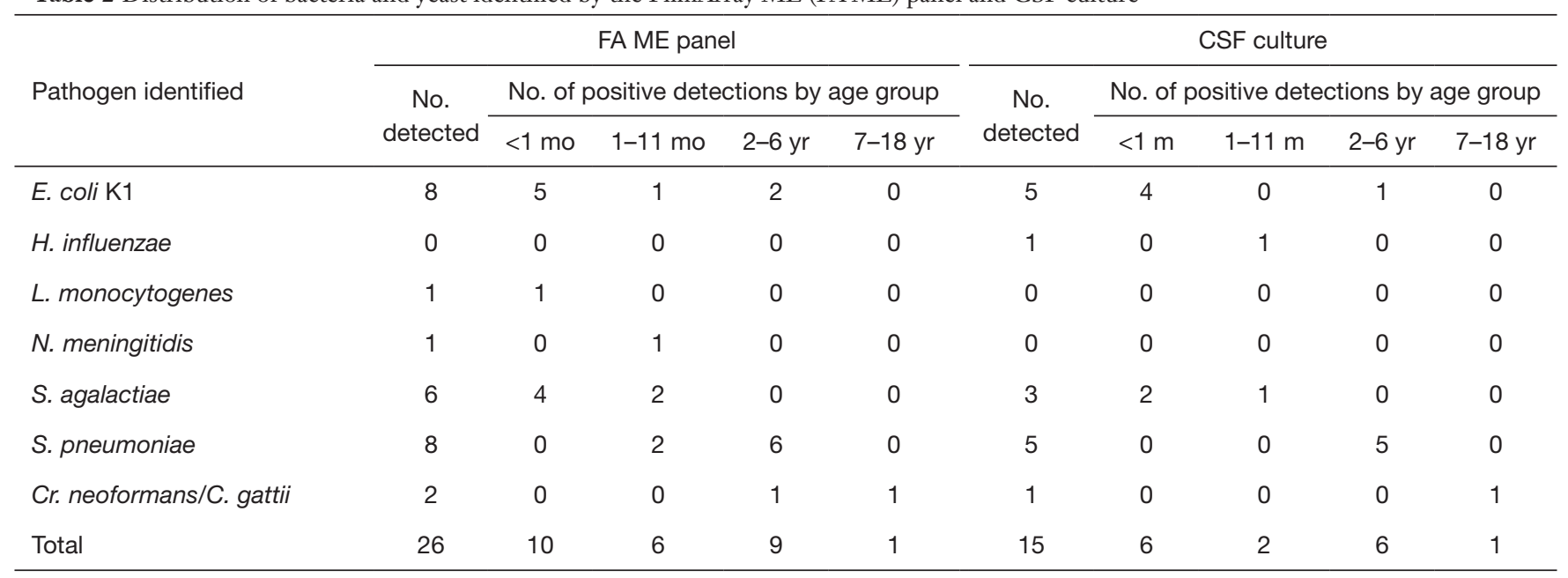

CSF, cerebrospinal fluid.

Table 3 Comparison of the positive and negative results in the FilmArray ME panel and comparator assays in bacteria and yeast detection

\begin{tabular}{|c|c|c|c|c|c|c|c|}
\hline Pathogen identified & \multicolumn{4}{|c|}{ No. of results } & \multicolumn{3}{|c|}{ Discordant results } \\
\hline E. coli K1 & 58 & 3 & 2 & 5 & 4 & 1 & 1 \\
\hline H. influenzae & 67 & 0 & 1 & 0 & NA & NA & NA \\
\hline L. monocytogenes & 67 & 0 & 0 & 1 & 1 & 0 & 0 \\
\hline S. agalactiae & 62 & 3 & 0 & 3 & 2 & 1 & 0 \\
\hline S. pneumoniae & 59 & 4 & 1 & 4 & 3 & 1 & 0 \\
\hline Cr. neoformans/C. gattii & 66 & 1 & 0 & 1 & 1 & 0 & 0 \\
\hline
\end{tabular}

C, CSF culture; F, FilmArray ME panel; P, Target PCR assay; +, positive result; -, negative result. NA, not applicable or not able to calculate.

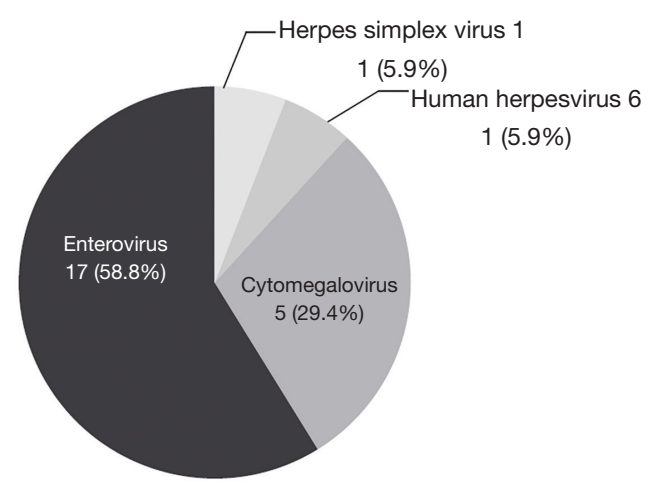

Figure 3 Virus detected by the FilmArray ME panel. ME, meningitis/encephalitis. culture, with a positive rate of $22.1 \%(15 / 68)$. For the FilmArray ME panel, 26 bacteria and fungi from 25 samples were detected, and the positive rate was 36.8\% (25/68) (Table 2). The FilmArray ME panel identified 14 pathogens in previously CSF culture-negative patients, while CSF culture identified pathogens in 4 of the 30 FilmArray ME panel-negative samples (Table 3). The most prevalent in the FilmArray ME panel detection were E. coli K1 (8/68, $11.8 \%)$, S. pneumoniae $(8 / 68,11.8 \%)$ and S. agalactiae $(6 / 68$ $8.8 \%)$. L. monocytogenes and $N$. meningitidis were detected in $1(1 / 68,1.5 \%)$ sample each, and C. neoformans/C. gattii was detected in $2(2 / 68,2.9 \%)$ samples. 
Table 4 Performance summary and characteristics of the FilmArray ME panel and CSF culture in bacteria and yeast detection

\begin{tabular}{lcccccc}
\hline Pathogen identified & OPA (\%) & Sensitivity (\%) & Specificity (\%) & PPV (\%) & NPV (\%) & Kappa \\
\hline E. coli K1 & 89.7 & 60 & 92.1 & 37.5 & 96.7 & 0.408 \\
H. influenzae & 98.5 & 0 & 100 & 0 & 98.5 & 0 \\
L. monocytogenes & 98.5 & NA & 98.5 & 0 & 100 & 0 \\
N. meningitidis & 98.5 & NA & 98.5 & 0 & 100 & 0 \\
S. agalactiae & 95.6 & 100 & 95.4 & 50 & 100 & 0.646 \\
S. pneumoniae & 92.7 & 80 & 93.7 & 50 & 98.3 & 0.577 \\
Cr. neoformans/C. gattii & 98.5 & 100 & 98.5 & 50 & 100 & 0.66 \\
\hline
\end{tabular}

OPA, overall percentage of agreement; PPV, positive predictive value; NPV, negative predictive value.

Among the FilmArray ME panel results, viral pathogens were detected in 17 samples, namely, EV (10 cases), CMV (5 cases), HSV-1 (1 case) and HHV-6 (1 case) (Figure 3). The patients' clinical data are reported in Table $S 2$.

\section{Correlation between the FilmArray ME panel and CSF culture in detecting bacteria and yeast}

In this study, a total of 476 individual FilmArray ME panel analyte tests were performed on 68 samples (for each sample, 6 bacterial and 1 fungal analyte tests were included: E. coli K1, H. influenzae, L. monocytogenes, N. meningitidis, S. pneumoniae, S. agalactiae and Cr. neoformans/C. gattii). The OPA between the FilmArray ME panel and CSF culture were $83.2 \%$ (396/476). For the individual target, the FilmArray ME panel had a relatively higher OPA than the CSF culture for the detection of bacteria and yeast, including $H$. influenzae, L. monocytogenes, $N$. meningitidis, S. agalactiae, and C. neoformans/C. gattii, while two analytes had lower sensitivities (89.7\% for E. coli K1 and $92.7 \%$ for S. pneumoniae) (Table 4). Using CSF culture as the diagnostic gold standard, the sensitivity and specificity of the individual FilmArray ME panel's components were calculated. The FilmArray ME panel demonstrated a sensitivity of $100 \%$ for 2 analytes: S. agalactiae and C. neoformans/C. gattii. The specificity was $92.1 \%$ or greater for all analytes. Comparison of FilmArray ME panel and CSF culture for E. coli K1, S. agalactiae, S. pneumoniae, and Cr. neoformans/C. gattii showed moderate agreement $(0.4<$ kappa $<0.7)$.

\section{Analysis of discrepant results}

The 19 samples with discrepant results are summarized in Table 5. There were 12 TP cases (E. coli K1: 4 cases;
S. agalactiae: 2 cases; $S$. pneumoniae: 3 cases; L. monocytogenes: 1 case; $N$. meningitides: 1 case; $C$. neoformans/C. gattii: 1 case) and $3 \mathrm{TN}$ cases (E. coli K1: 1 case; S. pneumoniae: 1 case; H. influenzae: 1 case) for the FilmArray ME panel results using comparator testing results (target PCR assay). The 1 $\mathrm{FN}$ case (E. coli $\mathrm{K} 1: 1$ case) and $3 \mathrm{FP}$ cases (E. coli K1: 1 case; $S$. agalactiae: 1 case; $S$. pneumoniae: 1 case) were determined with no additional evidence.

\section{Discussion}

As a major health problem in newborn infants and children worldwide, meningitis and encephalitis require early diagnosis and aggressive therapy $(11,12)$. However, similarities exist in patients with different pathogen infections in terms of clinical manifestations, making it difficult to diagnose meningitis and encephalitis with atypical clinical symptoms and signs. Due to the lack of rapid and reliable laboratory tests in etiological diagnosis, the rate of erroneous diagnosis is unacceptably high. Until recently, the etiology was unknown for approximately $50 \%$ of cases $(13,14)$, leading to a delay in the initiation of optimal treatment. Novel and fast molecular techniques help identify etiologies, prevent the use of unnecessary antibiotics and shorten the length of hospital stays (15). The BioFire FilmArray ME panel provides a comprehensive panel testing for 14 CNS pathogens simultaneously using a minimal amount of CSF with a rapid turn-around time (10). More recently, the use of the FilmArray ME panel for detecting pathogens has been reported to improve laboratory diagnosis $(16,17)$. To our knowledge, this study is the first report of the performance of the FilmArray ME panel in China, where we evaluated the potential clinical benefits in testing for various pathogens. 
Table 5 Discrepant investigation for samples with discordant results.

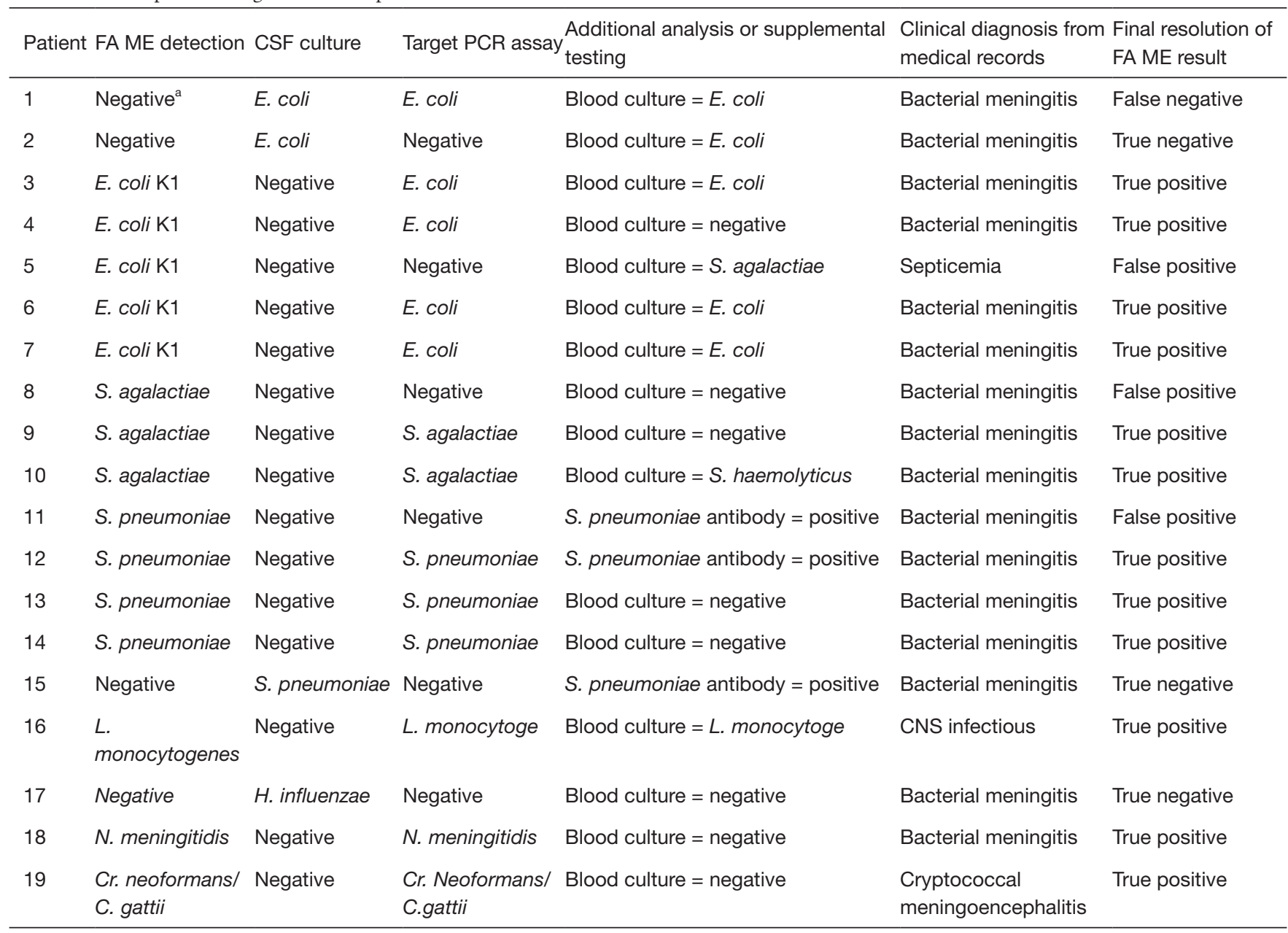

a, for E. coli, only the $\mathrm{K} 1$ capsular type is detected by the FilmArray ME panel.

In our study, demographic data analysis of patients revealed that there were more males than females $(67.6 \%$ vs. 32.4\%). This finding agreed with a study by Qazi et al., which showed that males were more significantly affected by bacterial meningitis than females ( $80 \%$ vs. $20 \%)(18)$. This difference may signify male dominance and sex discrimination in East Asia. A total of $51.5 \%(n=35)$ of our patients were below 1 year of age, showing that meningitis is more likely to occur in younger children than in older children. A study by Seth et al. confirmed that meningitis is most strongly and consistently associated with a young age, in which the majority of patients $(76 \%)$ were infants $<12$ months old (19).

The definitive diagnosis of bacterial meningitis has been historically based on culture, which has a sensitivity of $\leq 80 \%$ (20). As a gold standard for the diagnosis of meningitis, CSF culture was therefore used as the comparator assay in the present research. Previous studies have shown that CSF culture was positive in only $10 \%$ of antibiotic-pretreated patients in developing countries (21). Afifi et al. also found low rates of culture-positive CSF samples (8\%) in suspected cases of bacterial meningitis (22). In our study, 50 patients (73.5\%) had received antibiotic treatment before a LP was performed. Among these patients, only 10 (14.7\%) samples were positive in CSF culture, while 18 (26.4\%) samples were positive when detected with the FilmArray ME panel. In addition, the positive rate was influenced by the therapeutic time of antibiotics before LP. The detection number was lower with a longer use time of antibiotics in both methods. However, the FilmArray ME panel had relatively higher sensitivity than CSF culture when the use time of antibiotics was more than 1 day. Among 51 culturenegative CSF specimens, 14 were positive in the FilmArray ME panel detection. Wootton et al. confirmed that the 
FilmArray ME panel could enhance pathogen identification in CNS-infected patients with a negative Gram stain, and the panel detected pathogens not previously identified in $11(22.9 \%)$ of 48 patients (17). These findings show that the FilmArray ME panel can provide enhanced diagnosis in culture or Gram stain negative CSF specimens, especially after the administration of antimicrobial therapy.

Using FilmArray ME panel detection, this study identified bacteria and yeast in 25/68 patients (36.8\%). E. coli $\mathrm{K} 1$ and $S$. pneumoniae were the most common organisms detected, followed by S. agalactiae, C. Neoformans, L. monocytogenes and N. meningitidis. The FilmArray ME panel had a higher sensitivity than CSF culture in detecting almost all bacteria and yeast except $H$. influenzae. Furthermore, the association between age and pathogens was analyzed. In our study, E. coli $\mathrm{K} 1$ and $S$. agalactiae were the predominant pathogens inducing neonatal bacterial meningitis. The same result was reported by Arora et al., namely, that the FilmArray ME panel enhanced the identification of group B Streptococcus and E. coli in young infants with meningitis (16). Other studies in Australia, London and Canada also documented similar patterns, with $S$. agalactiae and E. coli being the major etiological agents for neonatal bacterial meningitis infection (23-25). $S$. pneumoniae was the predominant pathogen isolated in the 1-6 years age group, and this finding was consistent with a Korean study in which $S$. pneumoniae was the most detected etiologic pathogen beyond the neonatal period (26). Although mixed CNS infections are not uncommon in children, especially in immunocompromised individuals, infections with two or more pathogens are not easily detected by conventional methods. Hence, the biological significance of dual infections is currently not well understood. The FilmArray ME panel has the significant benefit of being able to identify coinfections. Five cases of dual infections were detected in our study, namely, a case of mixed bacterial-bacterial co-infection (E. coli $\mathrm{K} 1+$ $S$. pneumoniae), two cases of mixed bacterial-viral coinfection (S. pneumoniae + CMV, S. agalactiae + CMV), one case of mixed yeast-viral coinfection (C. neoformans $+\mathrm{CMV}$ ) and one case of mixed viral-viral coinfection (HHV-6 + CMV).

Any discrepancy between CSF culture and the FilmArray ME panel was analyzed in the present study. With regard to bacteria and yeast detection, the comparator targeted PCR assay and clinical data confirmed 12 of the positive results and 3 of the negative results detected by the FilmArray ME panel. For the $3 \mathrm{FP}$ results, the discrepancy investigation did not support the FilmArray ME panel results: an S. agalactiae
FP sample from a 1-month-old girl with normal CSF parameters; a $S$. pneumoniae FP sample from a 10-monthold girl, although the detection of anti-S. pneumoniae antibody was positive; and an E. coli $\mathrm{K} 1 \mathrm{FP}$ sample from a 26-day-old boy whose blood culture was shown to contain $S$. agalactiae. The false-positive results $(4.4 \%)$ with the FilmArray ME panel in our study were fewer than those reported in a previous study by Leber et al. (10), in which FP results accounted for $41 \%$ of bacterial results. Only an E. coli FN case was determined in a 20-day-old boy, and the reasons for the $\mathrm{FN}$ results were diverse. For E. coli, only the K1 capsular type was detected by the FilmArray ME panel, while other E. coli types also cause CNS infections. In addition, frozen samples or operational issues may produce negative results (27). Clinicians should be cautious when interpreting the results from the FilmArray ME panel, particularly with regard to FP results, which may lead to needless therapy and subsequent related drug toxicity (28).

There were several limitations in our study. First, we did not have viral comparative results to support the results detected by the FilmArray ME panel because a serological test is the only major available method to detect viruses in routine diagnosis in China (29). Moreover, the serological test results were not available for some samples in our study. Although many developed countries have used realtime PCR of the CSF for the daily detection of possible viruses (30), this assay was unavailable in our study. In contrast, the FilmArray ME panel fills the gap in virological testing in China. We identified 17 positive viral pathogens including EV $(\mathrm{n}=10), \mathrm{CMV}(\mathrm{n}=5), \mathrm{HSV}-1 \quad(\mathrm{n}=1)$ and HHV-6 ( $\mathrm{n}=1)$, among which only 3 samples were positive in viral serological tests. Second, the sample size was relatively small and likely had an impact on the statistical certainty of the FilmArray ME panel sensitivity and specificity calculations. Furthermore, since it was a retrospective study, selection bias is inevitable due to the criteria used for sample selection and the small volume of CSF tested.

\section{Conclusions}

This study demonstrated the capability of the FilmArray ME panel in the diagnosis of bacterial and fungal meningitis and therefore its potential use in facilitating enhanced patient care. The BioFire FilmArray ME panel may reduce diagnostic uncertainty in pediatric patients with suspected CNS infections, and its rapid diagnosis will enable optimization of antibiotic use. However, the FilmArray ME panel cannot identify some other pathogens 
not included in the panel (e.g., Mycobacterium tuberculosis, an important cause of meningitis in China), nor could it provide information about antibiotic susceptibilities. Thus, it should be noted that the FilmArray ME panel represents an adjunctive test rather than a replacement test.

\section{Acknowledgments}

We would like to thank the patients and their parents for their support in permitting us to publish the findings of our research.

Funding: This work was financially supported by the Love Charity Foundation Research Project in Shanghai Children's Medical Center (2017SCMC-AY004), the Key Developing Disciplines Project from Shanghai Municipal Commission of Health and Family Planning (2016ZB0104) and the Collaborative Innovation Center for Translational Medicine at Shanghai Jiao Tong University School of Medicine (TM201616).

\section{Footnote}

Conflicts of Interest: Y Xia was employed by company bioMérieux (Shanghai) Company Limited. The other authors have no conflicts of interest to declare.

Ethical Statement: The authors are accountable for all aspects of the work in ensuring that questions related to the accuracy or integrity of any part of the work are appropriately investigated and resolved. The study was approved by the Institutional Review Board and the Ethics Committee of Shanghai Children's Medical Center (SCMCIRB-K2017059). Written informed consent for specimen collection was obtained from the parents of the participants when lumbar puncture was carried out. But written informed consent for research could not be obtained as our study was a retrospective study.

\section{References}

1. Dong BQ, Tang ZZ, Lin M, et al. Epidemiologic surveillance for bacterial meningitis in 140000 children under 5 years of age in Nanning district, Guangxi province. Zhonghua Liu Xing Bing Xue Za Zhi 2004;25:391-5.

2. Li Y, Yin Z, Shao Z, et al. Population-based Surveillance for Bacterial Meningitis in China, September 2006December 2009. Emerg Infect Dis 2014;20:61-9.

3. Wang Y, Wang CQ, Wang XH. Etiology and antimicrobial susceptibility of children with bacterial meningitis. Available online: http://en.cnki.com.cn/Article_en/ CJFDTOTAL-SYQK200606020.htm

4. Le Saux N. Guidelines for the management of suspected and confirmed bacterial meningitis in Canadian children older than one month of age. Paediatr Child Health 2014;19:141-52.

5. Kanegaye JT, Soliemanzadeh P, Bradley JS. Lumbar puncture in pediatric bacterial meningitis: defining the time interval for recovery of cerebrospinal fluid pathogens after parenteral antibiotic pretreatment. Pediatrics 2001;108:1169-74.

6. Polage CR, Cohen SH. State-of-the-Art Microbiologic Testing for Community-acquired Meningitis and Encephalitis. J Clin Microbiol 2016;54:1197-202.

7. Banks JT, Bharara S, Tubbs RS, et al. Polymerase chain reaction for the rapid detection of cerebrospinal fluid shunt or ventriculostomy infections. Neurosurgery 2005;57:1237-43; discussion 1237-43.

8. Graf EH, Farquharson MV, Cárdenas AM. Comparative evaluation of the FilmArray meningitis/encephalitis molecular panel in a pediatric population. Diagn Microbiol Infect Dis 2017;87:92-4.

9. Messacar K, Breazeale G, Robinson CC, et al. Potential clinical impact of the film array meningitis encephalitis panel in children with suspected central nervous system infections. Diagn Microbiol Infect Dis 2016;86:118-20.

10. Leber AL, Everhart K, Balada-Llasat JM, et al. Multicenter Evaluation of BioFire FilmArray Meningitis/ Encephalitis Panel for Detection of Bacteria, Viruses, and Yeast in Cerebrospinal Fluid Specimens. J Clin Microbiol 2016;54:2251-61.

11. Kuti BP, Bello EO, Jegede TO, et al. Epidemiological, clinical and prognostic profile of childhood acute bacterial meningitis in a resource poor setting. J Neurosci Rural Pract 2015;6:549-57.

12. Briand C, Levy C, Baumie F, et al. Outcomes of bacterial meningitis in children. Med Mal Infect 2016;46:177-87.

13. Nigrovic LE, Fine AM, Monuteaux MC, et al. Trends in the management of viral meningitis at United States children's hospitals. Pediatrics 2013;131:670-6.

14. George BP, Schneider EB, Venkatesan A. Encephalitis hospitalization rates and inpatient mortality in the United States, 2000-2010. PLoS One 2014;9:e104169.

15. Soucek DK, Dumkow LE, VanLangen KM, et al. Cost Justification of the BioFire FilmArray Meningitis/ Encephalitis Panel Versus Standard of Care for Diagnosing Meningitis in a Community Hospital. J Pharm Pract 
2019;32:36-40.

16. Arora HS, Asmar BI, Salimnia H, et al. Enhanced Identification of Group B Streptococcus and Escherichia Coli in Young Infants with Meningitis Using the Biofire Filmarray Meningitis/Encephalitis Panel. Pediatr Infect Dis J 2017;36:685-7.

17. Wootton SH, Aguilera E, Salazar L, et al. Enhancing pathogen identification in patients with meningitis and a negative Gram stain using the BioFire FilmArray(®) Meningitis/Encephalitis panel. Ann Clin Microbiol Antimicrob 2016;15:26.

18. Qazi SA, Khan MA, Mughal N, et al. Dexamethasone and bacterial meningitis in Pakistan. Arch Dis Child 1996;75:482-8.

19. Seth R, Murthy PS, Sistla S, et al. Rapid and Accurate Diagnosis of Acute Pyogenic Meningitis Due to Streptococcus Pneumoniae, Haemophilus influenzae Type $\mathrm{b}$ and Neisseria meningitidis Using A Multiplex PCR Assay. J Clin Diagn Res 2017;11:FC01-4.

20. Neuman MI, Tolford S, Harper MB. Test characteristics and interpretation of cerebrospinal fluid gram stain in children. Pediatr Infect Dis J 2008;27:309-13.

21. Brouwer MC, Tunkel AR, van de Beek D. Epidemiology, diagnosis, and antimicrobial treatment of acute bacterial meningitis. Clin Microbiol Rev 2010;23:467-92.

22. Afifi S, Wasfy MO, Azab MA, et al. Laboratory-based surveillance of patients with bacterial meningitis in Egypt

Cite this article as: Du B, Hua C, Xia Y, Li J, Xie Y, Tao Y, Cao Q, Mo X. Evaluation of the BioFire FilmArray meningitis/ encephalitis panel for the detection of bacteria and yeast in Chinese children. Ann Transl Med 2019;7(18):437. doi: 10.21037/ atm.2019.08.103
(1998-2004). Eur J Clin Microbiol Infect Dis 2007;26:331-40.

23. Francis BM, Gilbert GL. Survey of neonatal meningitis in Australia: 1987-1989. Med J Aust 1992;156:240-3.

24. Heath PT, Nik Yusoff NK, Baker CJ. Neonatal meningitis. Arch Dis Child Fetal Neonatal Ed 2003;88:F173-8.

25. Stevens JP, Eames M, Kent A, et al. Long term outcome of neonatal meningitis. Arch Dis Child Fetal Neonatal Ed 2003;88:F179-84.

26. Cho HK, Lee H, Kang JH, et al. The causative organisms of bacterial meningitis in Korean children in 1996-2005. J Korean Med Sci 2010;25:895-9.

27. Lee CK, Chiu L, Yan G, et al. False negative results caused by erroneous automated result interpretation algorithm on the FilmArray 2.0 instrument. Clin Chem Lab Med 2018;56:e43-5.

28. Gomez CA, Pinsky BA, Liu A, et al. Delayed Diagnosis of Tuberculous Meningitis Misdiagnosed as Herpes Simplex Virus-1 Encephalitis With the FilmArray Syndromic Polymerase Chain Reaction Panel. Open Forum Infect Dis 2016;4:ofw245.

29. Zhao L, Zhou M, Wang B, et al. Clinical characteristics and outcome of clinically diagnosed viral encephalitis in southwest China. Neurol Sci 2015;36:2191-7.

30. Mutton K, Guiver M. Laboratory techniques for human viral encephalitis diagnosis. Infect Disord Drug Targets 2011;11:206-34. 


\section{Supplementary}

Table S1 Primers used in the PCR amplification

\begin{tabular}{lll}
\hline Primer & Forward & Reverse \\
\hline Bacteria-16S & AGAGTTTGATCMTGGCTCAG & TACGGYTACCTTGTTACGACTT \\
Fungus-26S & GCATATCAATAAGCGGAGGAAAAG & GGTCCGTGTTTCAAGACGG \\
E.coli-mdh & TGGTTGCTAACTGGAAAGGAAT & ACGTGTATTTGAAGCATTGCTG \\
s. pneumoniae-ply & CCCACTCTTCTTGCGGTTGA & TGAGCCGTTATTTTTCATACTG \\
S. agalactiae-cps & CAATCCTAAGTATTTCGGTTCATT & TAGGAACATGTTCATTAACATAGC \\
\hline
\end{tabular}

Table S2 Summary of clinical data on patients with positive viral pathogens confirmed by the FilmArray ME panel

\begin{tabular}{|c|c|c|c|c|c|c|c|}
\hline Patient & $\begin{array}{l}\text { Clinical diagnosis from } \\
\text { medical records }\end{array}$ & CSF cells & CSF protein & CSF glucose & CSF chlorides & Serological test & FA ME detection \\
\hline 1 & Purulent meningitis & 6929 & $3,529.9$ & 2.77 & 114.7 & - & CMV \\
\hline 2 & Viral encephalitis & 106 & 193 & 3.35 & 124.7 & - & EV \\
\hline 3 & Viral encephalitis & 1360 & 244 & 2.61 & 124.6 & - & EV \\
\hline 4 & Purulent meningitis & 500 & 418 & 2.85 & 120.4 & - & EV \\
\hline 5 & Viral encephalitis & 440 & 193 & 3.33 & 122.8 & - & EV \\
\hline 6 & Viral encephalitis & 300 & 1,273 & 1.76 & 121.8 & - & EV \\
\hline 7 & Viral encephalitis & 61 & 1,546 & 2.3 & 127 & HSV-1 IgM(-), IgG(-) & HSV-1 \\
\hline 8 & Purulent meningitis & 90 & 703 & 2.3 & 118 & - & EV \\
\hline 9 & CNS infection & 112 & 1,955 & 2.1 & 120 & - & EV \\
\hline 10 & Viral encephalitis & 0 & $<100$ & 3.3 & 127 & EV71 IgM( \pm$)$ & EV \\
\hline 11 & Viral encephalitis & 160 & 405 & 3.4 & 121 & - & EV \\
\hline 12 & Viral encephalitis & 600 & 235 & 2.9 & 122 & - & EV \\
\hline 13 & Viral encephalitis & 87 & 527 & 3.1 & 125 & - & CMV/HHV-6 \\
\hline
\end{tabular}

